\title{
Optimal Online-list Batch Scheduling
}

\author{
Jacob Jan Paulus ${ }^{a, *}$, Deshi Ye ${ }^{\mathrm{b}}$, Guochuan Zhang ${ }^{\mathrm{b}}$ \\ ${ }^{a}$ University of Twente, P.O. box 217, 7500AE Enschede, The Netherlands \\ ${ }^{\mathrm{b}}$ Zhejiang University, Hangzhou 310027, China
}

\begin{abstract}
We consider the online-list batch scheduling problem. Jobs arrive one by one and have to be assigned upon arrival to a scheduled batch such that the makespan is minimized. Each batch can accommodate up to $B$ jobs. We give a complete classification of the tractability of this online problem.
\end{abstract}

Key words: Batch Scheduling, Online Algorithms, Competitive Analysis

\section{Introduction}

In this paper we consider online-list scheduling on one batching machine. A set of jobs has to be scheduled on the batching machine which processes jobs parallel in batches. Each job $j$ is characterized by its processing time $p_{j}$. The batching machine has capacity $B$, which gives the maximum number of jobs that can be scheduled in a single batch. The processing time of a batch must be larger than or equal to the maximum processing time of all jobs in the batch. The objective is to minimize the makespan, that is the completion time of the last batch. Note that the order of the batches is of no importance, it does not influence the objective function, only the processing times of the created batches are of interest. The above type of batching is referred to as parallel batching or p-batch, contrary to an s-batch which processes jobs sequential with a start-up time for each batch [3]. The model of parallel batching finds applications in, for example, scheduling burn-in ovens used for circuit board manufacturing [8].

* Corresponding author.

Email address: j.j.paulus@utwente.nl (Jacob Jan Paulus). 
In the online-list version of this problem jobs from a sequence $\sigma$ are presented one by one to the scheduler. Upon arrival, the processing time of the job becomes known and the job has to be assigned immediately and irrevocably to a batch. The job is either included in a non-full existing batch (i.e. a batch with less than $B$ jobs assigned to it) or a new batch is created for this job. The processing time of each batch has to be fixed upon its creation, and a job $j$ can only be assigned to a batch with processing time at least $p_{j}$.

In the corresponding offline problem, the scheduler has all jobs available at $t=0$, and an optimal offline schedule can be found by applying the algorithm known as FBLPT (Full Batch Longest Processing Time) [7]. This algorithm schedules the $B$ jobs with largest processing time in the first batch, the next $B$ jobs with largest processing time in a second batch, etc.

For a sequence of jobs $\sigma$, we denote the makespan of the optimal offline schedule by $C^{*}(\sigma)$ and the makespan of the online schedule created by an online Algorithm $A$ by $C^{A}(\sigma)$. The performance of an online Algorithm $A$ is given by its competitive ratio defined as $\sup _{\sigma}\left\{C^{A}(\sigma) / C^{*}(\sigma)\right\}$. An online algorithm is called optimal if it has the smallest possible competitive ratio among all online algorithms.

In the literature only related problems have been studied. In [2] the onlinelist batching problem with the objective to minimize the average flow time is studied and an optimal 4-competitive algorithm is given. The considered model allows only to schedule the next job in the last created batch or to create a new batch, and the capacity of the batching machine is unlimited. Much more work has been done on the online-time version of the batching problem to minimize the makespan, where jobs arrive according to their release date. For the unlimited capacity case, optimal $(\sqrt{5}+1) / 2$-competitive algorithms were given in [6] and [12] and generalized in [10]. The tractability of the online-time problem has not yet been resolved for bounded capacity. The best known online algorithm is a 2-competitive algorithm for any capacity $B$ [11]. Only for the case $B=2$ a better algorithm is presented in [11], which is $7 / 4$ competitive. We refer to [9] for the more general problem with job families and a more extensive overview of the results on the online-time model.

The algorithms designed in this paper use what is called the "doubling" strategy. The idea behind this strategy is to use geometrically increasing batch processing times to approximate the optimal offline solution. However, as mentioned in [4], the increase is not always done by a factor of 2 . Online algorithms designed with this principle can be found, for example, in the literature on the problem of searching a line in the plane [1]. A short overview of other online problems solved with the "doubling" strategy can be found in [4].

This paper is organized as follows. Section 2 presents an optimal 4-competitive 
algorithm in case of unlimited capacity. Optimality follows from the known bounds for the online bidding problem, which is basically the same problem. The online bidding problem is a folklore online problem, for example, described in [5]. Section 3 deals with the bounded capacity case and contains the main contribution of the paper: For any given capacity $B$ we derive an optimal online algorithm. With these results, the tractability of online-list batch scheduling is settled.

\section{Unlimited capacity and online bidding}

In this section we consider the case of unlimited batch capacity and show an optimal 4-competitive algorithm. In case of unlimited batch capacity the optimal offline schedule has all jobs in one and the same batch of length equal to the largest processing time. So, the optimal offline makespan is given by $C^{*}(\sigma)=\max _{j \in \sigma}\left\{p_{j}\right\}$.

With unlimited batch capacity, the online-list batch scheduling problem is the same as the folklore online bidding problem, see e.g. [5]. The online bidding problem is stated as follows: An online player submits bids $b_{i}$ until it submits a bid larger than or equal to a threshold $T \geq 1$. The online player pays the sum of all submitted bids. It is not difficult to see that these problems are equivalent. The online scheduler determines a sequence of batch lengths $b_{1}<b_{2}<\ldots<b_{k-1}<b_{k}$ such that $b_{k-1}<p_{\max } \leq b_{k}$, and has makespan $\sum_{i=1}^{k} b_{i}$. Since the batch capacity is unlimited, no reasonable algorithm creates a batch for an arriving job that can be included in an existing batch. The online bidder determines a sequence of bids $b_{1}<b_{2}<\ldots<b_{k-1}<b_{k}$ such that $b_{k-1}<T \leq b_{k}$, and pays $\sum_{i=1}^{k} b_{i}$. Again, no reasonable bidder submits a bid smaller than the previous bid. The offline costs are $p_{\max }$ and $T$ for the scheduling and bidding problem, respectively.

To deal with this online problem we propose the following "doubling" algorithm.

\section{Algorithm $A^{\infty}$ :}

Schedule a job $j$ with processing time $p_{j} \in\left(2^{i-1}, 2^{i}\right]$ in a batch of length $2^{i}$. If no such batch exists, create it at the end of the current schedule.

The idea behind this algorithm is the same as behind all "doubling" type online algorithms. Even if the algorithm is forced to construct many different batches, we know that there exists a relatively long job compared to the total batch length. Informally, there is a growth rate of 2 in the batch lengths. This leads to the following performance guarantee of $A^{\infty}$. 
Theorem 1 For online-list batch scheduling with unlimited capacity, Algorithm $A^{\infty}$ is 4-competitive.

Proof: For any sequence of jobs $\sigma$, Algorithm $A^{\infty}$ creates for jobs with lengths in $\left(2^{i-1}, 2^{i}\right]$ at most one batch of length $2^{i}$. By normalizing, we let the smallest batch have length 2. Now, if the largest batch created for sequence $\sigma$ has length $2^{n}$, then

$$
C^{*}(\sigma)=\max _{j \in \sigma}\left\{p_{j}\right\}>2^{n-1}
$$

and

$$
C^{A^{\infty}}(\sigma) \leq \sum_{i=1}^{n} 2^{i}=2^{n+1}-2
$$

The competitive ratio of $A^{\infty}$ is bounded by

$$
\frac{C^{A^{\infty}}(\sigma)}{C^{*}(\sigma)}<\frac{2^{n+1}-2}{2^{n-1}}=4-\frac{1}{2^{n-2}}<4
$$

Thus, Algorithm $A^{\infty}$ is 4-competitive.

To show that Algorithm $A^{\infty}$ has the best possible competitive ratio, we consider one special adversary job sequence:

Definition The infinite job sequence $\sigma^{\text {adv }}$ has $p_{1}=1$ and each following job has length equal to the last created batch by the online algorithm plus a small amount $\epsilon>0$. The subsequence $\sigma_{k}^{\text {adv }}$ is given by the first $k$ jobs of sequence $\sigma^{\mathrm{adv}}$.

Job sequence $\sigma^{\text {adv }}$ depends on the online algorithm used, but any online algorithm must create a new batch for each new arriving job. Intuitively, this adversary is the strongest possible for both the bounded capacity and unlimited capacity case, meaning that it creates for any online algorithm the worst case instance. Therefore we will use this sequence of jobs throughout the paper to prove lower bounds on the competitive ratio and performance of online algorithms. With this adversary we have $p_{\max }=b_{k-1}+\epsilon$ if the sequence stops after job $k$. (In online bidding this means $T=b_{k-1}+\epsilon$.)

For online bidding, it is known that no bidding strategy is better than 4competitive [5]. This implies that no algorithm for online-list batch scheduling with unlimited capacity can have a competitive ratio less than 4 . However, for completeness of this paper, we include the proof of optimality of Algorithm $A^{\infty}$ and adopt it from [5].

Theorem 2 For online-list batch scheduling with unlimited capacity, no online algorithm is $(4-\delta)$-competitive, for any $\delta>0$.

Proof: Suppose there exists a $(4-\delta)$-competitive Algorithm $A$, and this al- 
gorithm is presented with job sequence $\sigma^{\text {adv }}$. For simplicity we denote the optimal offline makespan by $C_{k}^{*}$ and the online makespan of Algorithm $A$ by $C_{k}^{A}$ for the subsequence $\sigma_{k}^{\text {adv }}$. All what follows is subject to the small value $\epsilon$ in the construction of $\sigma^{\text {adv }}$, but by choosing $\epsilon$ appropriately small it does not effect the outcome. So, we choose $\epsilon$ small enough and leave it from the remainder of the analysis.

Define $\gamma_{k}=\frac{C_{k+1}^{A}}{C_{k}^{A}}$. The assumption on the competitiveness of Algorithm $A$ gives $C_{k+1}^{A} \leq(4-\delta) \cdot C_{k+1}^{*}$, which, by definition of $\sigma^{\text {adv }}$, can be rewritten as

$$
C_{k+1}^{A} \leq(4-\delta) \cdot\left(C_{k}^{A}-C_{k-1}^{A}\right)
$$

Dividing this inequality by $C_{k}^{A}$ gives a recursion on $\gamma_{k}$ :

$$
\gamma_{k+1} \leq(4-\delta) \cdot\left(1-\frac{1}{\gamma_{k}}\right)
$$

Since $1-\frac{1}{x} \leq \frac{x}{4}$, this implies $\gamma_{k+1} \leq(4-\delta) \cdot \frac{\gamma_{k}}{4}$. Thus $\gamma_{k} \leq\left(\frac{4-\delta}{4}\right)^{k} \gamma_{0}$, and so eventually $C_{k+1}^{A}<C_{k}^{A}$, which is a contradiction.

As a consequence of Theorem 2 we get that Algorithm $A^{\infty}$ is an optimal online algorithm.

\section{Bounded capacity}

In the previous section we have seen how the doubling strategy leads to an optimal algorithm if the batch capacity is unlimited. In this section we consider online-list batch scheduling with a fixed bounded capacity $B$ for each batch. To obtain an optimal algorithm for the bounded case, we have to use a different growth rate in batch lengths (different for each capacity $B$ ). However, the basic structure of the scheduling algorithm is the same as in Algorithm $A^{\infty}$.

Concrete, we propose the following online algorithm for the online-list batch scheduling problem with capacity $B$. If $B \leq 3$ we schedule the jobs greedily. If $B \geq 4$, we use a growth rate of $z_{B}$ in batch lengths instead of the rate 2 for the unlimited capacity case.

Algorithm $A^{B}$ :

If $B \leq 3$, then schedule a job $j$ with processing time $p_{j}$ in a non-full batch of length at least $p_{j}$. If no such batch exists, create a batch with length $p_{j}$ at the end of the current schedule.

If $B \geq 4$, then schedule a job $j$ with processing time $p_{j} \in\left(z_{B}^{i-1}, z_{B}^{i}\right]$ in a 
non-full batch of length $z_{B}^{i}$. If no such batch exists, create it at the end of the current schedule.

We choose $z_{B}$ such that

$$
z_{B}=\operatorname{argmin}_{x \geq 1}\left\{x+1+\frac{1}{x}+\frac{1}{x^{2}}+\ldots+\frac{1}{x^{B-2}}\right\},
$$

and show that the competitive ratio of Algorithm $A^{B}$ is

$$
\rho_{B}=\min _{x \geq 1}\left\{x+1+\frac{1}{x}+\frac{1}{x^{2}}+\ldots+\frac{1}{x^{B-2}}\right\}
$$

Before we determine the competitive ratio of $A^{B}$, we point out that $z_{B}$ and $\rho_{B}$ are unique for a given $B$. There is only one minimum in (1), since the derivative $1-\frac{1}{x^{2}}-\frac{2}{x^{3}}-\ldots-\frac{B-2}{x^{B-3}}$ is increasing in $x$ for $x \geq 1$. To indicate what kind of growth rates and competitive ratios we are dealing with, we display in Table 1 the values of $z_{B}$ and $\rho_{B}$ for some specific values of $B$.

Table 1

\begin{tabular}{|l|cccccccc|}
\hline $\mathrm{B}$ & 2 & 3 & 4 & 5 & 6 & 7 & 8 & $\infty$ \\
\hline$z_{B}$ & 1 & 1 & 1.5214 & 1.7614 & 1.8768 & 1.9349 & 1.9651 & 2 \\
$\rho_{B}$ & 2 & 3 & 3.6107 & 3.8344 & 3.9254 & 3.9651 & 3.9833 & 4 \\
\hline
\end{tabular}

Values of $z_{B}$ and $\rho_{B}$

Theorem 3 For online-list batch scheduling with capacity B, Algorithm $A^{B}$ is $\rho_{B}$-competitive.

Proof: For $B \leq 3$, we know that each batch in the online schedule contains at least one job with processing time equal to the length of the batch. So, by a load argument the offline makespan cannot be less than $\frac{1}{B}$ times the online makespan. Thus, Algorithm $A^{B}$ is $B$-competitive.

Consider $B \geq 4$. Let $\sigma$ be a worst-case instance for Algorithm $A^{B}$. By normalizing the job lengths let $z_{B}^{1}$ be the smallest online batch and $n$ such that $z_{B}^{n}$ is the largest online batch. Thus the online schedule consists of batches with lengths in $\left\{z_{B}^{1}, z_{B}^{2}, \ldots, z_{B}^{n}\right\}$. Note that for each $i$ there is at most one non-full batch of length $z_{B}^{i}$. In the following we derive three properties which we may assume for worst-case instance $\sigma$ :

(1) Each job $j$ scheduled in a batch of length $z_{B}^{i}$ has length $p_{j}=z_{B}^{i-1}+\epsilon$, with $\epsilon>0$ arbitrary small.

Decreasing the job lengths in a batch of length $z_{B}^{i}$ to $z_{B}^{i-1}+\epsilon$ does not affect the online makespan and may decreases the offline makespan. As in the proof of Theorem 2, we ignoring $\epsilon$ from now on. 
(2) For each batch length $z_{B}^{i}$, there is at most one batch.

If the worst case instance has more than $B$ jobs in batches of length $z_{B}^{i}$, then $B$ of these jobs are together in a batch in both the online and offline schedule. Due to property (1), removing these $B$ jobs causes a decrease of $z_{B}^{i}$ in the online makespan and a decrease of $z_{B}^{i-1}$ in the optimal offline makespan. Let $\tilde{\sigma}$ be the instance resulting by removal of these $B$ jobs from $\sigma$. Since $\sigma$ is a worst-case instance we have

$$
\frac{C^{A}(\sigma)}{C^{*}(\sigma)} \geq \frac{C^{A}(\tilde{\sigma})}{C^{*}(\tilde{\sigma})}=\frac{C^{A}(\sigma)-z_{B}^{i}}{C^{*}(\sigma)-z_{B}^{i-1}} .
$$

This implies that $z_{B} \cdot C^{*}(\sigma) \geq C^{A}(\sigma)$, and that the algorithm has competitive ratio of at most $z_{B}<\rho_{B}$. So, we only have to consider instances which result in an online schedule with for each batch length $z_{B}^{i}$ at most one batch.

(3) Each batch consists of only one job.

If the only batch of length $z_{B}^{i}$ contains $k$ jobs with $2 \leq k \leq B$, then we can remove $k-1$ of these jobs without decreasing the online makespan and possibly decrease the offline makespan.

By the above properties of $\sigma$, we get that the cumulative length of the $B$ largest batches in the online schedule is at most $z_{B}^{n}+z_{B}^{n-1}+\ldots+z_{B}^{n-B+1}$. By (1) this is equal to $\rho_{B} \cdot z_{B}^{n-1}$, that is $\rho_{B}$ times the largest offline batch. This argument can be repeated for the next $B$ largest batches in the online schedule. They are at most $\rho_{B}$ times the second largest offline batch, etc. Thus, Algorithm $A^{B}$ is $\rho_{B}$-competitive.

It remains to show the optimality of Algorithm $A^{B}$. For such a proof, the known results on online bidding are of no use since they need the unlimited capacity of the batches. More precisely, the change in the offline cost structure makes the comparison with online bidding impossible and complicates the analysis. Where the offline makespan is just $p_{\max }$ for the unlimited capacity case, we now have to consider the FBLPT solution. The next theorem, which we consider the main contribution of this paper, uses the structure of the FBLPT solution to prove that Algorithm $A^{B}$ is optimal. In fact, the next theorem also implies Theorem 2 by letting $B$ go to infinity.

Theorem 4 For online-list batch scheduling with capacity $B$, no online algorithm is $\left(\rho_{B}-\delta\right)$-competitive, for any $\delta>0$ and $B$.

Proof: Suppose there exists a $\left(\rho_{B}-\delta\right)$-competitive Algorithm $A$, and this algorithm is presented with job sequence $\sigma^{\text {adv }}$. Recall that due to the construction of $\sigma^{\text {adv }}$ each job has its own batch in the online schedule, regardless of the online algorithm used. As in Theorem 2, we choose the $\epsilon$ in the instance construction small enough to ignore it. Again, for simplicity we denote the optimal offline makespan by $C_{k}^{*}$ and the online makespan of Algorithm $A$ by $C_{k}^{A}$ for 
the subsequence $\sigma_{k}^{\text {adv }}$.

The optimal offline and online makespan can be expressed by

$$
\begin{aligned}
C_{k}^{*} & =p_{k}+p_{k-B}+p_{k-2 B}+\ldots \\
C_{k}^{A} & =p_{k+1}+p_{k}+\ldots+p_{2} \\
& =C_{k+1}^{*}+C_{k}^{*}+\ldots+C_{k-B+2}^{*}-C_{1}^{*} .
\end{aligned}
$$

Let $\gamma_{k}=\frac{C_{k+1}^{*}}{C_{k}^{*}}$, be the ratio between the value of two subsequent optimal offline makespans. Note that this is a different ratio from the one used in Theorem 2. Obviously the optimal offline makespan increases in $k$, thus $\gamma_{k} \geq 1$. By Algorithm $A$ being $\left(\rho_{B}-\delta\right)$-competitive, we have

$$
\begin{aligned}
\frac{C_{k}^{A}}{C_{k}^{*}} & =\frac{C_{k+1}^{*}+C_{k}^{*}+\ldots+C_{k-B+2}^{*}-C_{1}^{*}}{C_{k}^{*}} \\
& =\gamma_{k}+1+\frac{1}{\gamma_{k-1}}+\frac{1}{\gamma_{k-1} \gamma_{k-2}}+\ldots+\frac{1}{\gamma_{k-1} \gamma_{k-2} \ldots \gamma_{k-B+2}}-\frac{C_{1}^{*}}{C_{k}^{*}} \\
& \leq \rho_{B}-\delta .
\end{aligned}
$$

We assume $k$ to be large enough such that $\frac{C_{1}^{*}}{C_{k}^{*}} \leq \frac{\delta}{2}$, thus

$$
\gamma_{k}+1+\frac{1}{\gamma_{k-1}}+\frac{1}{\gamma_{k-1} \gamma_{k-2}}+\ldots+\frac{1}{\gamma_{k-1} \gamma_{k-2} \ldots \gamma_{k-B+2}} \leq \rho_{B}-\frac{\delta}{2}
$$

In the remainder of this proof we show that (2) and $\gamma_{k} \geq 1$ are contradicting. To obtain this contradiction, we introduce $\tilde{\gamma}_{k}:=\max \left\{\gamma_{k-1}, \ldots, \gamma_{k-B+2}\right\}$ and show that $\gamma_{k}<\tilde{\gamma}_{k}$ and $\tilde{\gamma}_{k}$ decreases below 1 .

By (2) and the definition of $\tilde{\gamma}_{k}$ we have

$$
\begin{aligned}
\rho_{B}-\frac{\delta}{2} & \geq \gamma_{k}+1+\frac{1}{\gamma_{k-1}}+\frac{1}{\gamma_{k-1} \gamma_{k-2}}+\ldots+\frac{1}{\gamma_{k-1} \gamma_{k-2} \ldots \gamma_{k-B+2}} \\
& \geq \gamma_{k}+1+\frac{1}{\tilde{\gamma}_{k}}+\frac{1}{\tilde{\gamma}_{k}^{2}}+\ldots+\frac{1}{\tilde{\gamma}_{k}^{B-2}} .
\end{aligned}
$$

Since $z_{B}$ minimizes $x+1+\frac{1}{x}+\frac{1}{x^{2}}+\ldots+\frac{1}{x^{B-2}}$ and the minimum is $\rho_{B}$, we have $\gamma_{k}<\tilde{\gamma}_{k}$. This can be seen by assuming $\gamma_{k} \geq \tilde{\gamma}_{k}$. The value of $\gamma_{k}$ can be decreased to $\tilde{\gamma}_{k}$ without violating (3). So, this would yield a better minimum for $x+1+\frac{1}{x}+\frac{1}{x^{2}}+\ldots+\frac{1}{x^{B-2}}$ than $z_{B}$ does. 
As a direct consequence of $\gamma_{k}<\max \left\{\gamma_{k-1}, \ldots, \gamma_{k-B+2}\right\}$, we get $\tilde{\gamma}_{k}<\tilde{\gamma}_{k-B+2}$. Now assume that $\tilde{\gamma}_{k}$ converges to some $y$. Then equation (2) holds when all $\gamma_{i}$ 's are substituted by $y$, implying that $y$ gives a better minimum in (1) than $z_{B}$ does. Thus, $\tilde{\gamma}_{k}$ cannot converge.

By the above we have that the value $\tilde{\gamma}_{k}$ is an upper bound on $\gamma_{k}$ and decreases below any fixed value. Thus, eventually $\gamma_{k}<\tilde{\gamma}_{k} \leq 1$, contradicting $C_{k+1}^{*} \geq$ $C_{k}^{*}$.

By Theorems 3 and 4, we obtain the optimality of online Algorithm $A^{B}$. From the proof of Theorem 4 we see that any optimal online algorithm presented with $\sigma^{\text {adv }}$ must behave like Algorithm $A^{B}$ as $k$ grows large. No matter which optimal algorithm is used, the upper bound $\tilde{\gamma}_{k}$ must converge to $z_{B}$. In order to let $\tilde{\gamma}_{k}$ converge to $z_{B}$ the value $\gamma_{k}$ must converge to $z_{B}$. Therefore, as $k$ grows large the batch size has growth rate $z_{B}$.

\section{Concluding remarks}

This paper presents an optimal online algorithm for online-list batch scheduling with any batch capacity $B$. For $B \leq 3$ this algorithm is a greedy type algorithm, i.e. each batch has the same length as the first job scheduled in it. As $B$ goes to infinity the growth rate $z_{B}$ in the online algorithm goes to 2 and its competitive ratio $\rho_{B}$ to 4 . Therefore, the known results for the unlimited capacity case are implied by the new results for the bounded capacity case.

\section{Acknowledgments}

We thank Johann Hurink for helpful remarks. Part of this research was done while the first author visited Zhejiang University, Hangzhou. He is grateful for the hospitality received.

Part of this research has been funded by the Dutch BSIK/BRICKS project, NSFC (60573020), NSFC (10601048) and Chinese 973 project (2007CB310900).

\section{References}

[1] R.A. Baeza-Yates, J.C. Culberson, and G.J.E. Rawlins. Searching in the plane. Information and Computation, 106(2):234-252, 1993. 
[2] W.W. Bein, L. Epstein, L.L. Larmore, and J. Noga. Optimally competitive list batching. Lecture Notes in Computer Science (Algorithm Theory - SWAT2004), 3111:77-89, 2004.

[3] P. Brucker. Scheduling Algorithms. Springer-Verlag, fourth edition, 2004.

[4] M. Chrobak and C. Kenyon. Competitiveness via doubling. SIGACT News, $37(4): 115-126,2006$.

[5] M. Chrobak, C. Kenyon, J. Noga, and N.E. Young. Oblivious medians via online bidding. Lecture Notes in Computer Science (LATIN 2006), 3887:311$322,2006$.

[6] X. Deng, C.K. Poon, and Y. Zhang. Approximation algorithms in batch processing. Journal of Combinatorial Optimization, 7(3), 2003.

[7] C.Y. Lee and R. Uzsoy. Minimizing makespan on a single batch processing machine with dynamic job arrivals. International Journal on Production Research, 37(1):219-236, 1999.

[8] C.Y. Lee, R. Uzsoy, and L.A. Martin-Vega. Efficient algorithms for scheduling semiconductor burn-in operations. Operations Research, 40(4):764-775, 1992.

[9] Q. Nong, J. Yuan, R. Fu, L. Lin, and J. Tian. The single-machine parallelbatch on-line scheduling problem with family jobs to minimize makespan. International Journal of Production Economics, 111(2):435-440, 2008.

[10] C.K. Poon and W. Yu. A flexible on-line scheduling algorithm for batch machine with infinite capacity. Annals of Operations Research, 133(1):175-181, 2005.

[11] C.K. Poon and W. Yu. On-line scheduling algorithms for a batch machine with finite capacity. Journal of Combinatorial Optimization, 9(2):167-186, 2005.

[12] G. Zhang, X. Cai, and C.K. Wong. On-line algorithms for minimizing makespan on batch processing machines. Naval Research Logistics, 48(3):241-258, 2001. 